\title{
A CROSS SECTIONAL STUDY ON THE QUALITY OF LIFE AFTER ABLATIVE SURGERY OF HEAD AND NECK CANCER IN A CAUCASIAN POPULATION
}

K.Y. Dong, M.S. Chan, S.T. Ong. A cross sectional study on the quality of life after ablative surgery of head and neck cancer in a Caucasian population. Annal Dent Univ Malaya 2002; 9: 1-6.

\section{ABSTRACT}

Twenty-one patients who underwent ablative surgery for head and neck cancer at Bristol Dental Hospital and School, England between the years 1996 and 2002 were enrolled in the study. A self-completed questionnaire based on Head and Neck Specific measures was addressed to the patients.

The overall post-operative quality of life among these patients is acceptable. About half of the patients complained of moderate to severe difficulty in opening mouth wide (55\%) and dry mouth $(45 \%)$ and about one-third had major problems in swallowing solid food $(36 \%)$, sleep disturbance $(32 \%)$, trouble eating and enjoying meals (32\%) and speech problems (32\%). The study shows a tendency for the quality of life to improve steadily with increasing post-operative interval.

It is hoped that the results will provide an insight into the patients' functional and psychological recovery, which will in turn help to facilitate the planning of appropriate strategies to improve their quality of life.

Key words: quality of life, head and neck cancer, Caucasians, surgery.

\section{INTRODUCTION}

The two main goals of cancer therapy are to achieve a disease-free physical state of health $\&$ an acceptable functional recovery after treatment. Oral cancer and its current treatment modalities such as surgery, radiotherapy or a combination of both may expose a patient to considerable physiological, psychological and social strains, due to problems in oral functional status and physical appearance. Patients may suffer from various degrees of orofacial disturbances caused by pain, dryness of the mouth, inadequate prosthetic rehabilitation and limitation of speech and deglutition. This is despite major advances in microvascular technique in intra-oral reconstruction (1-3).

A patient who is cured of cancer but as a result of the treatment will never be able to eat, speak or look normal again has a reduced quality of life $(\mathrm{Q} o \mathrm{~L})$. Whilst quality of life forms an important consideration in the management of patients with cancer, it is difficult to define. It has been described as a person's
Original Article

K.Y. Dong ${ }^{1}$, M.S. Chan ${ }^{2}$, S.T. Ong

${ }^{1,2}$ Dental Student
${ }^{3}$ Associate Professor
${ }^{3}$ Department of Oral and Maxillofacial Surgery
Faculty of Dentistry
University of Malaya
50603 Kuala Lumpur
Tel:79674807
Fax: 79674534
Corresponding author - Assoc. Prof. Ong Siew Tin

sense of well being that stems from satisfaction or dissatisfaction with the areas of life that are important. However it is difficult to measure, can be long and distressing and at best gave an approximate assessment of a patient's detailed individual symptoms (4). Rogers et al. (4) also reported that it is difficult to identify studies and questionnaires previously reported that dealt with quality of life assessment in patients with oral cancer. There are nonetheless some studies that evaluated functional disturbances and measured the quality of life after definitive treatment for head and neck cancers (5-16). The head and neck specific measures developed by the European Organisation for Research and Treatment of Cancer (EORTC) were validated by Bjordal and co-workers $(17,18)$. The module comprises 30 questions addressing symptoms, function and psychosocial aspects, and 5 items of yes/ no fields on aspects of analgesic, supplemental feeding and weight.

The aim of this study is to identify the postoperative problems faced by the oral cancer patients using a self-assessment questionnaire based on the European Organisation for Research and Treatment of Cancer (EORTC) Head and Neck specific (H\&N35) model. It is hoped that the results will provide an insight into the patients' functional and psychological recovery, which will in turn help to facilitate the planning of appropriate strategies to improve their quality of life.

\section{MATERIALS AND METHODS}

The intended subjects were patients who had ablative surgery for oral cancer (with or without radiotherapy). These patients were identified when they attended their review appointment in May 2002, in the Primary Care 
Unit, Bristol Dental School and Hospital, England. The patients were given the Patients Information Sheet prior to signing the consent form for participation in the study. Patients who were physically or mentally challenged and patients with severe hearing or language problems not associated with surgery were not included. The study was given ethical approval by the South West Local Research Ethics Committee.

Demographic details were recorded and surgical parameters such as type and site of tumour, date of surgery and mode of reconstruction were also noted.

A self-completed questionnaire with 29 questions was addressed to the patients. This questionnaire was adapted from the EORTC-H\&N35. Because the target group of this study were oral cancer patients, questions specific to the throat and sexuality were omitted. The questionnaire thus contained 25 questions to assess the severity of the problems faced by the patients in speech, mastication, deglutition, physical appearance and psychological well being. This was followed by four questions about usage of analgesia, nutritional supplements, feeding tube and adjuvant radiotherapy. This information may be additionally useful to indicate the patients' current status.

The questionnaire was explained and the patients were instructed to answer all the questions by rating the severity of each condition as score 1 (not at all), score 2 (a little), score 3 (quite a bit) or score 4 (very much). The total score for each patient was calculated. The maximum value of severity obtainable for scoring the quality of life in this study is 100 . A score of 25 would indicate an excellent post-operative quality of life while a score of 100 indicated very poor quality of life. Between these extremes, a score of below 50 is good while higher scores would indicate a significant reduction in the quality of life.

The quality of life is also analyzed in terms of functional disturbance in the different domains listed in the questionnaire. A score of 3 or 4 is to be taken as a significant disturbance.

\section{RESULTS}

A total of 22 patients were recruited during the study period of eight weeks. One patient who had surgical excision of adenoid cystic carcinoma of the parotid gland was excluded from the analysis in view of the totally extra-oral involvement. All patients were Caucasians. The patients had all been treated between the years 1996 and 2002 . Out of the 21 patients, 12 (57.1\%) were male and $9(42.9 \%)$ were female. The mean age was 61.3 years (range of $36-88$ ) on completion of this questionnaire (Figure 1). The distribution of the patients by sites of tumour was as shown in Table 1. Eighty-one percent of the patients $(n=17)$ were treated with surgery alone and $19 \%$ $(n=4)$ with surgery followed by radiotherapy.

Twenty patients $(95.2 \%)$ were treated for squamous cell carcinoma, while the remaining one patient $(4.8 \%)$ for adenoid cystic carcinoma. More than $90 \%(90.5 \% ; n=19)$ of the patients were free of recurrence at the time of evaluation while $9.5 \%(n=2)$ had tumour recurrence. The average postoperative interval was 21.8 months with a range of $1-75$ months. Closure of the intraoral defects was accomplished by primary closure in $19 \%(n=4)$ and by reconstruction in $81 \%(n=17)$ of the cases. Fifty nine percent had intraoral soft tissue reconstructions which included 9 cases of free radial forearm flap and one each of pectoralis major flap and buccal pad of fat. Three cases of mandibular resection had reconstruction with bone grafts while the two patients with maxillectomy had the defects restored with prostheses.

Table 2 represents the total score on quality of life (QoL) and surgical parameters. None of the patients scored 25 (excellent). Fifteen of the patients (71.4\%) scored 50 or below which indicates a reasonably good QoL. The remaining seven patients $(28.6 \%)$ gave scores that indicated significant reduction in QoL. Figure 2 shows the mean total QoL score against postoperative interval. The mean QoL scores were calculated by taking the average of the total scores of all patients within the same group. There was a reduction in the scores for QoL up to 4-year review.

The high QoL score at 4-5 year review was rated by an 88 years old woman who was unable to tolerate

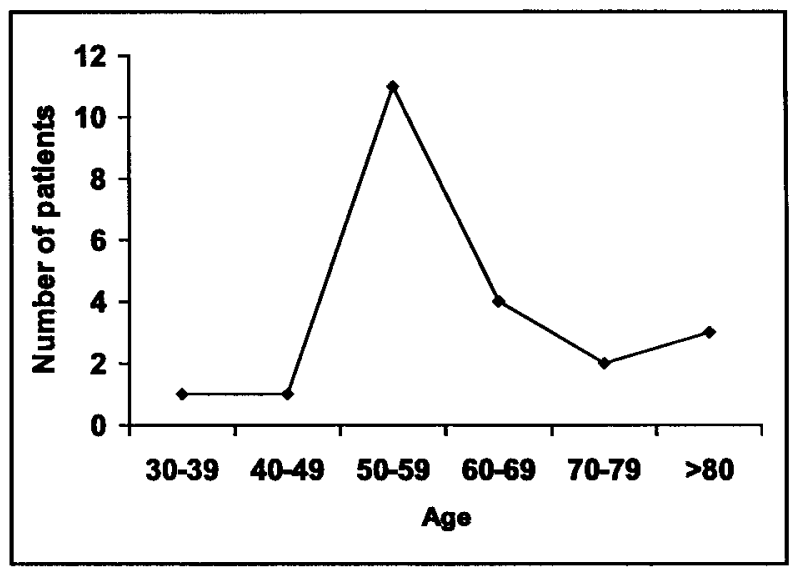

Figure 1: Age distribution of patients

Table 1. Site distribution of primary tumour $(n=21)$

\begin{tabular}{ccccccccc}
\hline Anterior 2/3 of tongue & Floor of mouth & Alveolus & Soft palate & Tonsillar fossae & Cheek & Lip & Maxillary antrum \\
\hline 6 & 3 & 4 & 2 & 2 & 2 & 1 & 1 \\
\hline
\end{tabular}


Table 2. Total Score for the questionnaire against Surgical Parameters of all the patients (The table is arranged with the scores in ascending order)

\begin{tabular}{|c|c|c|c|c|c|c|c|c|}
\hline No & I.D. & Age & $\begin{array}{c}\text { Site of primary } \\
\text { tumour }\end{array}$ & $\begin{array}{l}\text { Mode of } \\
\text { reconstruction }\end{array}$ & RT & Rec & $\begin{array}{l}\text { POI (In } \\
\text { months) }\end{array}$ & $\begin{array}{l}\text { Total } \\
\text { score }\end{array}$ \\
\hline 1 & A. & 61 & Tongue & Radial FF & - & - & 41 & 26 \\
\hline 2 & B. & 55 & Tonsillar Fossae & Radial FF & - & $Y$ & 29 & 26 \\
\hline 3 & c. & 56 & Tonsillar Fossae & - & - & - & 17 & 26 \\
\hline 4 & D. & 69 & Lip & - & - & - & 10 & 27 \\
\hline 5 & E. & 80 & Cheek & Buccal Fat Pad & - & - & 10 & 29 \\
\hline 6 & F. & 58 & Tongue & Radial FF & - & - & 19 & 30 \\
\hline 7 & G. & 76 & Tongue & Radial FF & - & - & 42 & 31 \\
\hline 8 & H. & 54 & Alveolus & RoMC & - & - & 1 & 33 \\
\hline 9 & 1. & 61 & FOM & Radial FF & - & - & 44 & 40 \\
\hline 10 & J. & 36 & Tongue & - & - & - & 2 & 41 \\
\hline 11 & K. & 55 & Cheek & - & - & - & 3 & 43 \\
\hline 12 & L. & 84 & Tongue & Radial FF & - & - & 43 & 43 \\
\hline 13 & M. & 58 & FOM & Radial FF & $Y$ & $\mathbf{Y}$ & 75 & 46 \\
\hline 14 & N. & 66 & Premaxilla & Silicone Facial Prostheses & - & - & 36 & 49 \\
\hline 15 & o. & 56 & Alveolus & RoMC & - & - & 2 & 50 \\
\hline 16 & P. & 56 & Soft Palate & Radial FF & - & - & 4 & 52 \\
\hline 17 & Q. & 71 & FOM & Radial FF & $Y$ & - & 12 & 54 \\
\hline 18 & $\mathbf{R}$ & 52 & Soft Palate & Radial FF & - & - & 24 & 57 \\
\hline 19 & S. & 54 & Maxillary Antrum & Obturator for maxillae & $Y$ & - & 5 & 63 \\
\hline 20 & $\mathbf{T}$ & 58 & Tongue & Pectoralis Major Flap & - & - & 5 & 65 \\
\hline 21 & U. & 88 & Alveolus & RoMC & - & - & 50 & 74 \\
\hline
\end{tabular}

$\begin{array}{ll}\text { - } Y=\text { Yes } & \text { - FF = Free Flap } \\ \text { - RT = Radiotherapy } & \text { - FOM = Floor of Mouth } \\ \text { - Rec = Recurrence } & \text { RoMC = Restoration of Mandibular Continuity } \\ \text { - } P O I=\text { Postoperative Interval } & \end{array}$

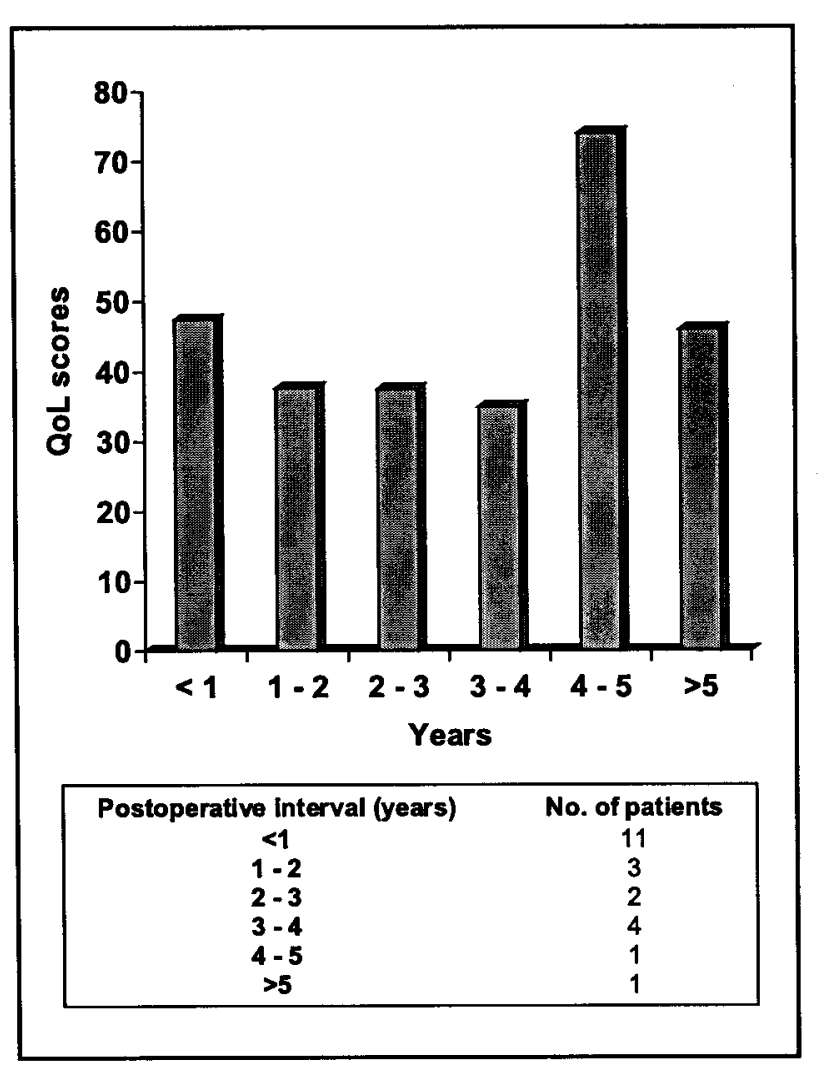

Figure 2: Mean Score for quality of life QoL against post-operative interval the complete dentures, and she had severe difficulties in mastication, swallowing and speech. As for the patient who had first surgery more than 5 years prior to the survey, he had multiple tumours on the head and neck region with recurrence after the ablative surgery and was undergoing radiotherapy during the past week.

Table 3 shows the distribution of the prevalent problems faced by the patients during the past week. About $29 \%(n=6)$ considered their employment status not significantly affected mainly because they were retired and their retirement bore no relation to their cancer treatment. The most prominent problems were dry mouth and limitation of mouth opening, while almost one third had difficulty enjoying meals and swallowing solid food. Fourteen percent $(n=3)$ were bothered by their appearance to a greater or lesser extent while about one third reported having major sleep disturbances. About $19 \%(n=4)$ of them considered their emotion to be significantly affected by the cancer treatment since they suffered from lack of self-confidence and some level of fear and anxiety. In addition, about $43 \%(n=9)$ of patients had used painkillers while one third of them reported to have taken nutritional supplements other than vitamins to improve their general health. Just $14 \%(n=3)$ of patients used the feeding tube and only $10 \%(n=2)$ had adjuvant radiotherapy in the past week. 
Table 3. Frequency (\%) of patients who scored $3 \& 4$ (i.e. quite a bit and very much) in severity, in the different domains (The table is arranged with the frequency in ascending order)

\begin{tabular}{ll}
\hline Questions & $\begin{array}{c}\text { Percentage } \\
\text { of patients }\end{array}$ \\
\hline
\end{tabular}

During the past week, have you

Felt ill? 0

Had trouble having social contact with family?

Had problems swallowing liquid?

Had trouble having social contact with friends?

Had trouble having physical contact with other people?

Had problems swallowing pureed food?

Had trouble eating in front of your family?

Had trouble going out in public?

Felt bothered by your physical appearance?

Had problems with your teeth?

Had lost of weight?

Had fear and anxiety?

Had lack of self-confidence?

Had problems with sense of smell \& taste?

Had pain in your mouth/jaw?

Had lost of appetite?

Had trouble eating in front of other people?

$\mathrm{Had}$ problems in your work/career?

Had trouble eating?

Had trouble enjoying your meals?

Had trouble talking to other people?

Had sleep disturbance?

Had problems swallowing solid food?

Had a dry mouth?

Had problems opening your mouth wide?

\section{DISCUSSION}

Determining the quality of life of head and neck cancer patients would mean assessing the oral function status, physical well being, psychological and social status of the patients (11). Some studies measured quality of life after ablative surgery of head and neck cancer based on professional assessment $(6,19,20)$. However, it is now generally accepted that a questionnaire completed by the patient is a more accurate and sensitive tool. Several studies that assess the quality of life using a self-completed questionnaire were identified $(4,11,12)$. There were also some studies that used both methods to measure the quality of life $(10,21)$.

Two-third of the patients in this survey rated a quality of life that was reasonably good. However, it is neither possible to generalize nor compare between patients, given that there were few common surgical parameters. It would be even more unlikely to compare with other studies. However, an analysis of the disturbance in various aspects could be done. About two third of the patients reported to be unaffected or mildly affected in most of the domains except for difficulty in opening the mouth wide and dry mouth for which about half of the them suffered moderate to severe difficulty. About two thirds of the patients felt that their overall oral functions in eating, enjoying meals, swallowing solid food and talking were not really affected postoperatively. A majority of them did not have much problem in eating in front of their family and other people. Almost everyone was able to swallow liquid and pureed food. This was probably the result of a carefully planned reconstruction after surgical removal of oral cancer, as about $77 \%$ of the patients had some form of reconstruction to the intraoral soft and/or hard tissues and oral rehabilitation by means of dentures, implants and other prosthesis. Factors such as extensive loss of functional soft tissues, problems with dentition, adjuvant radiotherapy, absence of reconstruction, difficulty in tolerating full dentures and old age could contribute to moderate and severe difficulty in mastication, speech and deglutition. Dental status is important, as patients tend to be limited to semi-solid diets postoperatively and have difficulty wearing dentures $(7,8)$.

Oral function such as speaking is often compromised after ablative surgery of the tongue. Total glossectomy obviously leaves many functional deficits and reconstruction could only improve articulatory function to some extent (13). In this study, one patient had a total glossectomy reconstructed with pectoralis major flap. She reported severe limitations in mastication and deglutition with moderate difficulty in speech and scored high in these domains. Vaughan (21) reported that the functional results using the radial forearm graft are superior to myocutaneous flap repairs and recommended the technique strongly. There being only one patient for comparison, it is unreliable to establish any relationship between the modes of reconstruction with the oral functions.

Most patients claimed that they did not lose weight as they had no loss of appetite and were able to eat without much difficulty during the past week. None of the patients generally felt ill during the past week. This is quite reasonable given that during the survey, they were free of disease and in good psychophysical condition.

Almost half of the patients used painkillers although only about one-fifth reported experiencing moderate to severe pain in the mouth/jaw. This could be due to the possibility that some patients might be using the painkillers to relieve pains in other regions of the body that was not related to the ablative surgery.

All patients reported that they do not face any problems in having social contact with the family as the family members have been able to accept and adapt to the patients' altered physical and emotional conditions after the surgical removal of cancer. Nonetheless, there was a minority of patients who did have major trouble in the social and physical contact with other people apart from their family members. The reasons could mainly be due to the altered physical appearance and the disability they faced after the ablative surgery. About a quarter of the patients felt that their employment status were significantly affected 
after the surgery as the cancer treatment did reduced their ability to perform optimally in their daily work. The rest of the patients were retired at the time of diagnosis and the retirement was not related to the cancer treatment.

Only about $15 \%$ of the patients complained that their physical appearance was moderately to severely altered. Different individuals cope with altered body image in different manners, resulting in a variation in perception of the physical appearance. Presumably most patients were grateful to be cured of the cancer and therefore the importance of body image was secondary to the first priority in the treatment of the cancer.

Attention to psychological issues has been increasing in services for people who have cancer. Although head and neck cancer make up only about $4 \%$ of all cancers, it might be predicted that psychological distress would be common in this population. From the psychology point of view, some patients did suffer from some level of psychological disturbances. As a result, one third reported suffering from sleep disturbances whereas one fifth had lack of self-confidence, fear and anxiety. Anxiety and depression were strongly associated with poor function in most domains $(22,23)$. Patients with impaired function, disfigurement and pain have a high possibility of an associated depressive disorder as impaired oral function could induce depression and anxiety and these could trigger problems with oral function too.

The overall QoL score was reduced (i.e. quality of life improves) in the patients whose postoperative intervals was between 3 and 4 years compared to those less than a year (Figure 5). Being a cross sectional study with a small sample, it is not possible to induce if quality of life improves gradually after one year postoperatively. Roger et al (24) however found that the quality of life domain scores were similar between the one-year and long-term groups suggesting that a person's response at one year following surgery is a useful indicator of the long-term success. Rehabilitation by means of speech therapy, physical therapy, social service and rehabilitation nursing could also help to improve the quality of life after ablative surgery (5). Another factor that could help in improving the quality of life post operatively is the style of coping that could influence the functional status of patients with oral cancer. The total score on quality of life of the patient with postoperative interval between 4 and 5 years could be age related, with its attending inability to cope with oral changes and prosthesis. As for the patient with the postoperative intervals of more than 5 years, his rating a higher score compared to the mean score for the group less than a year is understandable given his recent radiotherapy treatment.

This study only reflects the individual quality of life postoperatively, and no comparison could be made with the preoperative status. In addition, preoperative data was difficult to obtain retrospectively. Insufficient data in the tumour staging, defect location and size caused some difficulty in grouping the patients into various categories that would otherwise facilitate comparison with the results of other studies.

\section{CONCLUSION}

Within the limits of this study, the data shows that following ablative surgery for oral cancers, most patient continue to enjoy a reasonably quality of life. Majority of the patients had quite a bit of problems with limitation of mouth opening and dry mouth besides having difficulty with taking solid food. The favourable scores in the various functional domains may reflect on the successful reconstruction of the surgical defect. However, a longitudinal study would be necessary to establish this relationship. Future longitudinal studies into these aspects, including a preoperative assessment, would be helpful to gain insight into the patients' functional and psychological problems and assist in their rehabilitation.

\section{ACKNOWLEDGEMENT}

This report was extracted from an eight-week elective project undertaken by the first author at the Primary Care Unit at Bristol Dental Hospital and School, United Kingdom.

The authors acknowledge and thank the following persons for the unqualified assistance and cooperation given to the first author, during her elective attachment at the said hospital.

1. Professor J G Cowpe, the Head of Division and Professor of Oral Surgery, Bristol Dental Hospital and School; who was the overseas supervisor

2. Staff of the Primary Care Unit at Bristol Dental Hospital and School especially Mr. P G Guest, Consultant Maxillofacial Surgeon, Mr Ceri Hughes and Mrs Sarah Constant for their invaluable assistance in patient selection

3. Mrs Theresa Munns, Administrator of Dental Clinical Dean's Office, Department of Oral and Dental Science for her administrative contribution

4. Patients of the Joint Head and Neck Clinic, Bristol Dental Hospital and School, who willingly participated in this survey.

\section{REFERENCES}

1. Schliephake H, Neukam FW, Schmelzeisen R, et al. Long-term quality of life after ablative intraoral tumour surgery. J Craniomaxillofac Surg 1995; 23: $243-9$.

2. Netscher DT, Meade RA, Goodman CM, Alford EL, Stewart MG. Quality of life and disease- 
specific functional status following microvascular reconstruction for advanced (T3 and T4) oropharyngeal cancers. Plast Reconstr Surg 2000; 105(5): 1628-34.

3. Vaughan ED, Bainton R, Martin IC. Improvements in morbidity of mouth cancer using microvascular free flap reconstructions. J Craniomaxillofac Surg 1992; 20: 132-4.

4. Rogers SN, Fisher SE, Woolgar JA. A review of quality of life assessment in oral cancer. Int J Oral Maxillofac Surg 1999; 28: 99-117.

5. Olson ML, Shedd DP. Disability and rehabilitation in head and neck cancer patients after treatment. Head Neck Surg 1978; 1: 52-8.

6. Teichgraeber J, Bowman J, Goepfert H. New test series for the functional evaluation of oral cavity cancer. Head Neck Surg 1985; 8: 9-20.

7. Finlay PM, Dawson F, Robertson AG, et al. An evaluation of functional outcome after surgery and radiotherapy for intraoral cancer. $\mathrm{Br} \mathrm{J}$ Oral Maxillofac Surg 1992; 30: 14-7.

8. Moroi HH, Okimoto K, Terada Y. The effect of an oral prosthesis on the quality of life for head and neck cancer patients. J Oral Rehab 1999; 26: 265-73.

9. Haribhakti VV, Kavarana NM, Tibrewala AN. Oral cavity reconstruction: an objective assessment of function. Head Neck 1993; 15: 119-24.

10. Bundgaard T, Tandrup O, Elbrond O. A functional evaluation of patients treated for oral cancer. A prospective study. Int J Oral Maxillofac Surg 1993; 22: 28-34.

11. Schliephake H, Neukam FW, Schmelzeisen, Varoga B, Schneller H. Long Term quality of life after ablative intraoral tumour surgery. $\mathrm{J}$ Cranio Maxillofac Surg 1995; 23: 243-9.

12. Schliephake H, Rufferd $K$, Schneller $T$. Prospective study of the quality of life of cancer patients after intraoral tumour surgery. J Oral Maxillofac Surg 1996; 54: 664-9.

13. Bjordal K, Ahlner-Elmqvist M, Hammerlid E, et al. A prospective study of quality of life in head and neck cancer patients. Part II: Longitudinal data. Laryngoscope 2001; 111(8): 1440-52.

14. Wilson KM, Rizk NM, Armstrong SL, et al. Effects of hemimandibulectomy on quality of life. Laryngoscope 1998; 108: 1574-7.
15. Pauloski BR, Logemann JA, Colangelo LA, et al. Surgical variables affecting speech in treated patients with oral and oropharyngeal cancer. Laryngoscope 1998; 108: 908-16.

16. Schliephake H, Schmelzeisen R, Schonweiler R, et al. Speech, deglutition and life quality after intraoral tumour resection. A prospective study. Int J Oral Maxillofac Surg 1998; 27: 99-105.

17. Bjordal K, Ahlner-Elmqvist $\mathrm{M}$, Tollesson E et al. Development of a European Organisation for Research and Treatment of Cancer (EORTC) questionnaire module to be used in quality of life assessments in head and neck cancer patients. Acta Oncol 1994; 33: 879-85.

18. Bjordal K, Kaasa S. Psychometric validation of the EORTC core quality of life questionnaire, 30-item version and a diagnostic-specific module for head and neck cancer patients. Acta Oncol 1992; 31: 311-21.

19. Rogers SN, Lowe D, Patel M, et al. Clinical function after primary surgey for oral and oropharyngeal cancer: an 11-item examination. $\mathrm{Br}$ J Oral Maxillofac Surg 2002; 40: 1-10.

20. Chen C, Zhang Z, Gao S, et al. Speech after partial glossectomy: a comparison between reconstruction and nonreconstruction patients. J Oral Maxillofac Surg 2002; 60: 404-7.

21. Konstantinovic VS. Quality of life after surgical excision followed by radiotherapy for cancer of the tongue and floor of the mouth: evaluation of 78 patients. J Cranio-Maxillofac Surg 1999; 27: 1927.

22. Vaughan ED. The radial forearm free flap in orofacial reconstruction: personal experience in 120 consecutive cases. J Cranio-Maxillofac Surg 1990; 18: 2-7.

23. Hassanein KAAM, Musgrove BT, Bradbury E. Functional status of patients with oral cancer and its relation to style of coping, social support and psychological status. Br J Oral Maxillofac Surg 2001; 39: 340-5.

24. Rogers SN, Hannah L, Lowe D, et al. Quality of life 5-10 years after primary surgery for oral and oropharyngeal cancer. J Cranio-Maxillofac Surg 1999; 27: 187-91. 